\title{
A Cloud Model to Parameterize Convection
}

\author{
A. A. Barker AND W. R. Kininmonth \\ Commonwealth Meteorology Research Centre, Melbourne, Victoria, Australia
}

(Manuscript received 29 June 1973, in revised form 12 September 1973)

\begin{abstract}
This paper describes a parameterization of convection developed for use with a multi-level primitive equation model of the atmosphere. This parameterization is similar in form to that of Kuo, who postulates that boundary layer air rises moist adiabatically before mixing with the large-scale flow. A major difference from Kuo arises, however, when we allow condensate (the cloud material) to be evaporated to the largescale flow during the mixing process. This parameterization conserves moisture and energy of the largescale flow in a fashion similar to Manabe, and is suitable for general circulation studies as well as shortterm prediction.

The parameterization tends to adjust the vertical profile of temperature to the pseudo-adiabatic lapse rate from the boundary layer, and the relative humidity tends to saturation. Adjustments are made frequently to points in the tropics. Results are compared with a similar integration using Manabe's convective adjustment scheme. The parameterization presented maintains stable integration, and has certain advantages when compared to the Manabe parameterization. The new parameter introduced, the maximum amount of cloud material at any level, can be obtained from cloud model results and may also be measured directly.
\end{abstract}

\section{Introduction}

At the present time, a good deal of attention is being paid to subgrid-scale processes for use in general circulation models. (An excellent summary of the present state of the art is presented in the GARP Publication Series No. 8.) In this paper we concentrate on a parameterization of convection which retains enough simplicity for use in general circulation models, while at the same time providing a more physically realistic representation of convection. First we shall briefly review the various parameterizations of convective activity.

The parameterizations used to include moist convection in numerical models of the atmosphere can be broadly described in three categories:

(i) Boundary-layer convergence of moisture as the source of latent heat was initially developed by Charney and Eliassen (1964).

(ii) Integrated convergence of moisture as a measure of cloudiness was postulated by Kuo (1965).

(iii) Manabe et al. (1965) maintained stability of the model atmosphere by a lapse rate adjustment.

These parameterizations and their later developments, are extensively discussed by Bates (1972) and Ogura (1972). Briefly the parameterizations and their limitations are as follows:

1) Boundary layerlatent heat release for the column in terms of the vertical motion at the top of the boundary layer. The scheme can be kept simple in form, and assuming saturation at the top of the boundary layer, moisture need not be explicitly predicted within the model framework, although more sophisticated schemes of this type have been presented by Ooyama (1969) and Rosenthal (1970). Using a multi-level model of the atmosphere, the major problem with this parameterization is to describe the distribution of released latent heat in the vertical. An adiabatic assumption dictates that most latent heat is released in the lower troposphere, which is in contrast to observations (e.g., Yanai, 1963) that the atmospheric heating appears in the high troposphere.

2) The convergence of moisture into a volume by large-scale advection through the troposphere and by evaporation from the surface can be related to the rate of formation of new cloud. As the clouds decay and mix with their environment, the properties of the clouds are diffused to the broad-scale flow. Kuo (loc. cit.) considered pseudo-adiabatic ascent of boundary-layer air within the cloud and subsequent mixing of the temperature and moisture excess to the broad-scale flow. All condensate was considered to leave the system as precipitation. In this model most of the sensible heat resulting from the latent heat release is realized in the upper troposphere where the temperature excess of the pseudo-adiabatic updraft is a maximum. The cooling observed in the lower and middle troposphere of tropical disturbances as described by Riehl (1969) and Zipser (1969) is not accounted for by this model. 


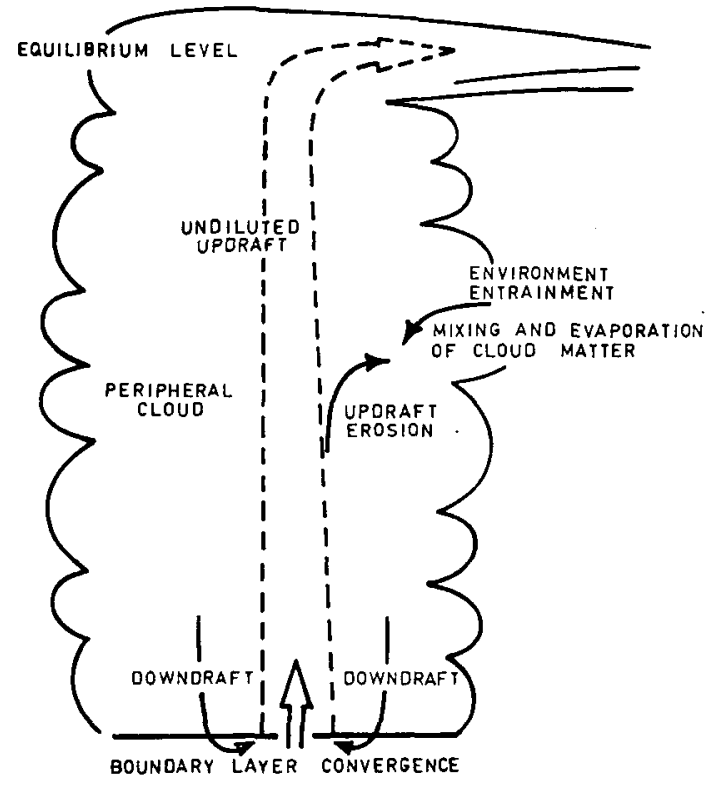

FIG. 1. Schematic representation of cloud model.

3) Manabe's (loc. cit.) and Arakawa et al.'s (1968) mathematical approach of lapse rate adjustment returns saturated unstable layers to a pseudo-adiabatic profile, while conserving energy and moisture. Not only does this scheme carry out a prime function of convection, namely the maintenance of a stable atmosphere, but also shows many of the characteristics observed in the atmosphere. The lapse rate adjustment cools the lower layer and heating appears in the upper layer. With surface heat and moisture flux operating in the numerical model this energy is rapidly pumped to higher levels. A disadvantage of the scheme is the sudden changes of temperature and mixing ratio which occur when a conditionally unstable environment is brought to saturation. Benwell and Bushby (1970) and Krishnamurti and Moxim (1971) have proposed criteria for reducing this initial thermodynamic shock. However, the situation still occurs where only restricted layers in the column need to be adjusted. This can lead to locally unrealistic temperature and moisture profiles, although the zonal average may be quite satisfactory.

A parameterization to include the effect of subsidence heating by the locally compensating downdrafts in the clear air has been outlined and empirically tested by Pearce and Riehl (1968). The data used suggested that clear air subsidence, on the average, counted for little of the vertical energy transport in regions of deep convection.

Recent observational studies which emphasize the convective aspect have been made by Reed and Recker (1971), Nitta (1972) and Bunker (1971). These observed results can be used in conjunction with simple models of convection to derive bulk properties of cumulus cloud populations (Yanai et al., 1972; Ogura and Cho, 1973). Further, the observational results allow for a reasonable qualitative comparison with results obtained from convective parameterizations in a general circulation model. There is little work comparing the various parameterizations of convection, either between parameterizations or with the observations. A recent exception is a paper by Elsberry and Harrison (1972) who compare the convective parameterization schemes of Kuo, Pearce and Riehl, and Rosenthal. The differences between these schemes when used on real data emphasize the need for more physically realistic parameterizations of convection.

\section{The cloud model}

As a basis for our model we shall assume that "hot towers" are dominant in the modification of the largescale flow. The concept of ascent in hot towers was proposed by Riehl and Malkus (1958) to describe the vertical energy transport in the equatorial trough zone. A few thousand of these hot towers at any time transport the boundary air to the high troposphere with the cores being protected by a peripheral cloud mass.

The model is illustrated in Fig. 1. Boundary-layer air rises moist adiabatically in an undiluted updraft. It is assumed that the updraft condensate is distributed in a definite profile, and contributes to precipitation and evaporation. The peripheral cloud mass is consumed by entrainment of unsaturated environment air, with consequent evaporation of cloud matter. The peripheral cloud mass must be continually replenished by erosion from the updraft. We consider a cloud ensemble to be statistically in a steady state, and to occupy a small fraction of the grid area.

The model implicitly incorporates downdrafts. There is some uncertainty as to whether it is broad-scale descent of clear air, or localized downdrafts within the cloud, which contribute most to supplementing the boundary-layer convergence. In practice, both probably contribute; however, in this model we assume downdrafts in the cloud as the only component reinforcing the boundary-layer convergence. These downdrafts are formed from convergence of moisture in upper levels, where an integrated moisture convergence is computed in a similar manner to Kuo (loc. cit.). This integrated moisture is redistributed between the updraft vapor and updraft condensate. It is important to realize that this implicitly assumes that middle tropospheric air descends to the boundary layer where it supplements boundarylayer convergence in the formation of the updraft. The manner in which mass, moisture and energy are conserved, and how the model redistributes them, will become more apparent in Section 3.

In an earlier study using this model Kininmonth (1970) demonstrated that the evaporation of condensate could consume sufficient heat during the outward mixing of updraft air that the heat excess of the buoyant updraft is negated. Under this condition the vicinity of 
the cloud is cooler than the broadscale flow. The updraft condensate at any level $\left(\sigma_{u}\right)$ is an important parameter in the model as it indirectly determines the heat loss to the flow by evaporation of cloud matter. A profile has been determined by Kininmonth (loc. cit.) for a tropical continental environment. The profile is reproduced in Fig. 2 and compared with the hydrometeor profiles as determined by the numerical cloud models of Squires and Turner (1962), Weinstein (1968), and Ogura and Takahashi (1971). The profile given by Ogura and Takahashi represents a developing storm cloud, and is presented to give some idea of the variance possible in $\sigma_{w}$. Direct measurement of cloud hydrometeor contents in cumulus towers has proved difficult (see Warner, 1970).

\section{The numerical parameterization}

The parameterization is incorporated into a multilevel primitive equation model with an explicit leap-frog time step. Temperature $T(k)$ and mixing ratio $q(k)$ are model variables at level $k$. We will calculate the change in $T$ and $q$ profiles, and the precipitation produced at a grid point over two time increments when convection occurs.

We assume the parameterization is initiated within the model if two conditions are satisfied: if the profile is conditionally unstable, and if there is boundary-layer convergence plus a net convergence of water vapor over the unstable layers. The first condition is a requirement for convection. Synoptic-scale convergence in the boundary layer has been discussed by Malkus (1963) as a requirement for the development of hot towers.

When the conditions of convection are satisfied, the efflux of heat and moisture from the updraft $(u)$ to the large-scale flow or environment $(e)$, are related to the rate of mass efflux (b) from the updraft. Because of the difficulty in specifying the dependence of mass efflux on height, we shall assume for simplicity that $b$ is constant with height. The theory extends directly to the case where $b$ is height-dependent. At any level applying the equation for eddy transfer (see Appendix A), the rate of change of $T$ and $q$ are given by

$$
\left.\begin{array}{l}
\frac{\partial T}{\partial t}=b\left[\left(T_{u}-T_{e}\right)-\frac{L}{C_{p}} \sigma_{u}^{*}\right] \\
\frac{\partial q}{\partial t}=b\left[\left(q_{u}-q_{e}\right)+\sigma_{u}{ }^{*}\right]
\end{array}\right\} .
$$

Here $\sigma_{u}{ }^{*}$ is that part of the updraft condensate concentration evaporated to the environment at the level, $L$ the latent heat of phase change, and $C_{p}$ the specific heat of air at constant pressure.

In (1) the environment profile is readily obtained from the grid-point data of the numerical model, while the updraft profile is calculated as a moist adiabat from the boundary layer. The updraft condensate profile is

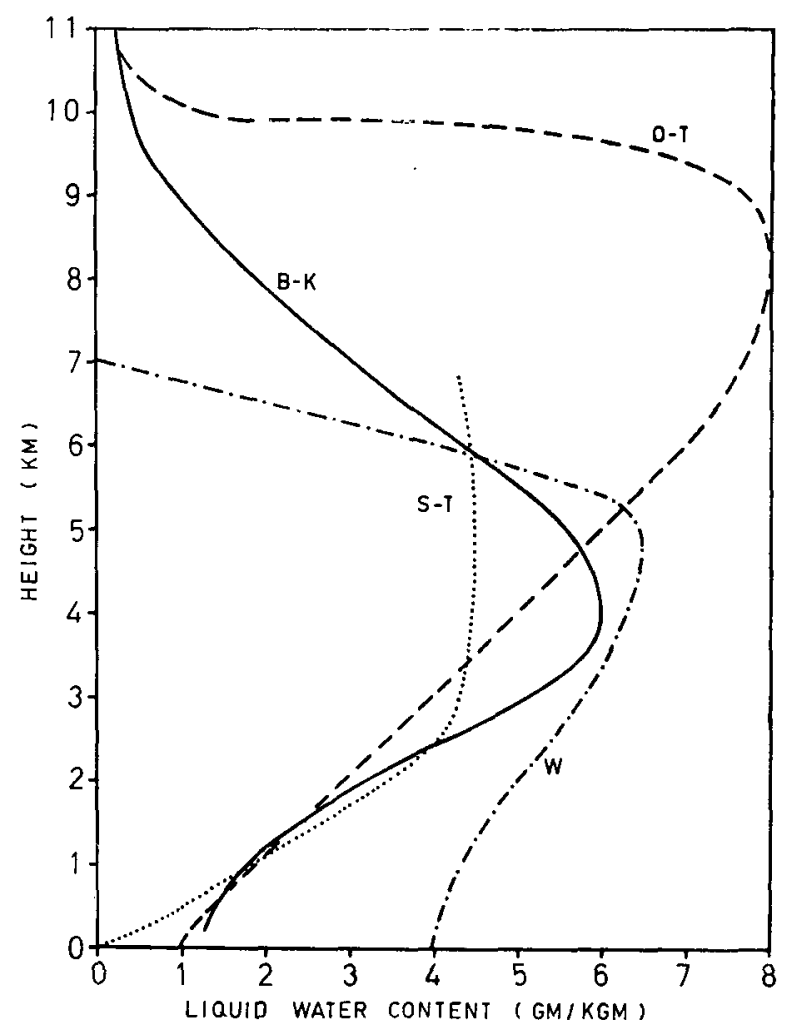

FIG. 2. Total updraft hydrometeor profile $\left(\mathrm{gm} \mathrm{kg}^{-1}\right)$ as uscd for the $(\mathrm{B}-\mathrm{K})$ model, compared with profiles obtained from other cloud models [Squires and Turner (S-T), Ogura and Takahashi $(\mathrm{O}-\mathbf{T})$, and Weinstein $(\mathrm{W})]$.

taken as the profile determined by Kininmonth. That part of the condensate evaporated to the environment, $\sigma_{u}{ }^{*}$, is obtained from the updraft condensate concentration $\sigma_{u}$, as a function of the environment relative humidity $h$ (see Appendix B), i.e.,

where

$$
\sigma_{u}^{*}=a \sigma_{u}
$$

$$
a=\left\{\begin{array}{lll}
0, & \text { for } h \geqslant 1.0 \\
1-\frac{h-0.5}{0.5}, & \text { for } & 0.5<h<1.0 \\
1, & \text { for } h \leqslant 0.5 .
\end{array}\right.
$$

An estimate of the mass efflux rate $b$ is made based on the conservation of moisture. According to the cloud model, moisture is converged in the updraft, and condensate and vapor are dispersed horizontally from the updraft. Condensate either falls to the ground as precipitation or is evaporated to the environment.

The rate of moisture convergence in the updraft (call it AMC) during a time interval 2st (where $2 \delta t=\tau+1-\tau-1)$ is obtained from the rate of increase of moisture due to large-scale processes:

$$
\mathrm{AMC}=\int_{p(B)}^{p(T)} \frac{\left(q^{\tau+1}-q^{\tau-1}\right)}{2 \delta t} \frac{d p}{g},
$$


TAJLE 1. Model levels.

\begin{tabular}{ccc}
\hline \hline Level & $Q^{*}$ & $\begin{array}{c}Z \\
(\mathrm{~km})\end{array}$ \\
\hline 1 & 0.0040 & 37.5 \\
2 & 0.0129 & 29.5 \\
3 & 0.0234 & 25.6 \\
4 & 0.0361 & 22.8 \\
5 & 0.0512 & 20.5 \\
6 & 0.0694 & 18.5 \\
7 & 0.0911 & 16.8 \\
8 & 0.1171 & 15.2 \\
9 & 0.1482 & 13.7 \\
10 & 0.1855 & 12.3 \\
11 & 0.2301 & 10.9 \\
12 & 0.2836 & 9.55 \\
13 & 0.3475 & 8.20 \\
14 & 0.4241 & 6.75 \\
15 & 0.5158 & 5.35 \\
16 & 0.6256 & 3.9 \\
17 & 0.7570 & 2.4 \\
18 & 0.9143 & 0.85 \\
Surface & 1.0000 & 0 \\
& &
\end{tabular}

* $Q(k)=p(k) / p_{*}$, where $p_{*}$ is the surface pressure.

where $p(B)$ is the pressure of the cloud base, $p(T)$ the pressure at the cloud top, $q^{\tau+1}$ the mixing ratio at timestep $\tau+1$ due to large-scale processes above, and $g$ the gravitational acceleration.

The rate of moisture increase due to convection (call it CMC) follows from the vertical integration of (1):

$$
\mathrm{CMC}=\int_{p(B)}^{p(T)} b\left(\sigma_{u}{ }^{*}+q_{u}-q_{e}\right) \frac{d p}{g} .
$$

From (2) and the definition of $\sigma_{u}$ the rate of precipitation follows as

$$
\mathrm{PPT}=\int_{p(B)}^{p(T)} b\left(\sigma_{u}-\sigma_{u}^{*}\right) \frac{d p}{g} .
$$

By combining (4) and (5) the total efflux of moisture from the updraft is obtained. This is equal to the largescale convergence of moisture for moisture to be conserved; hence,

$$
\mathrm{AMC}=\int_{p(B)}^{p(T)} b\left(\sigma_{u}+q_{u}-q_{e}\right) \frac{d p}{g} .
$$

For simplicity it is assumed that the rate $b$ of mass efflux with height is constant above the cloud base; thus, from (3) and (6), we have

$$
b=\frac{\int_{p(B)}^{p(T)}\left(q^{\tau+1}-q^{\tau-1}\right) \frac{d p}{g}}{\int_{p(B)}^{p(T)}\left(\sigma_{u}+q_{u}-q_{e}\right) \frac{d p}{g}} .
$$

Thus, the rate of mass efflux from the model clouds with condensate profiles $\sigma_{u}$ is determined by the amount of moisture advected into the grid area.
With both $b$ and $\sigma_{u}{ }^{*}$ defined, it is possible to compute the rate of convective energy change (CEC) during the interval as

$$
\mathrm{CEC}=\frac{C_{p}}{g} b \int_{p(B)}^{p(T)}\left[\left(T_{u}-T_{e}\right)-\frac{L}{C_{p}} \sigma_{u}{ }^{*}\right] d p .
$$

But the rate of energy change due to large-scale processes (AEC) is given by

$$
\mathrm{AEC}=\frac{C_{p}}{g} \frac{\int_{p(B)}^{p(T)}\left[T_{e}^{\tau+1}-T_{e}^{\tau-1}\right] d p}{2 \delta t} .
$$

In principle, these rates of energy change can be equated with the rate of latent heat release (LHR) from precipitation by the relationship

$$
\mathrm{AEC}+\mathrm{LHR}=\mathrm{CEC} \text {. }
$$

The use of the assumptions $\bar{W}=0$, the neglect of subsidence, the implicit inclusion of downdrafts which do not interact with the boundary layer, and equal mass efflux from the updraft at all levels, lead to an error in the computation of CEC. A correction is applied to $\mathrm{CEC}$ in the form of a small constant temperature change $\delta T$, at all levels, where

$$
\delta T=\frac{[\mathrm{AEC}+\mathrm{LHR}-\mathrm{CEC}]}{\frac{C_{p}}{g} \int_{p(B)}^{p\left(T^{(}\right)} d p} .
$$

This correction term is considered further in the discussion of the results.

The final temperature and mixing ratio at each level resulting from the interaction of the convection with the large-scale flow follows from (1) as

$$
\left.\begin{array}{c}
T^{\tau+1}(k)=T_{e}^{\tau-1}(k)+b\left[T_{u}(k)-T_{o}(k)\right. \\
\left.-\frac{L}{C_{p}} \sigma_{u}^{*}(k)\right] 2 \delta t+\delta T \\
q_{e}^{\tau+1}(k)=q_{e}^{\tau-1}(k)+b\left[q_{u}(k)-q_{e}(k)+\sigma_{u}^{*}(k)\right] 2 \delta t
\end{array}\right\} .
$$

Thus, the convective processes are seen to affect the parameter changes at a grid point, but the convection processes are controlled by the large-scale flow.

\section{Calculations}

The parameterization was incorporated into an 18level $N-20$ hemispheric general circulation model which has been run to quasi-equilibrium. The model was developed by Hunt (1972, personal communication) and is based on a previous model of Manabe and Hunt 
(1968). Hunt uses the moist lapse rate adjustment scheme of Manabe et al. (loc. cit.) to allow for convective processes. The convective parameterization outlined here in Section $3(\mathrm{~B}-\mathrm{K})$ replaces the scheme due to Manabe (M).

Initial integration with the $\mathrm{B}-\mathrm{K}$ scheme indicated that procedural difficulties occurred in several areas-first in the calculation of the updraft temperature $T_{u}(k)$ and mixing ratio $q_{u}(k)$. The model levels are in the $Q$-coordinate system, where $Q(k)=p(k) / p_{*}$ ( $p_{*}$ being the surface pressure), and details are presented in Table 1. The lowest level is at $0.85 \mathrm{~km}$, and this does not allow an accurate representation of the boundarylayer air which forms the updraft. The best representation appeared to be obtained from the anemometer level values given by

$$
\left.\begin{array}{rl}
T_{a} & =0.6 T_{e}(18)+0.4 T_{s} \\
q_{a} & =0.6 q_{e}(18)+0.4 q_{s}
\end{array}\right\}
$$

where the subscripts $a$ and $s$ refer to anemometer and surface levels, respectively, and the surface air is assumed to be saturated. The updraft air, with properties $T_{a}, q_{a}$, is considered to rise dry adiabatically until it is saturated, and then moist adiabatically. Hence, if $q_{a}$ is greater than the saturation mixing ratio $q_{\text {sat }}$ at $T_{a}$, the updraft profile rises from a base $T_{u}=T_{a}$. However, if there is a saturation deficit $\left[q_{\mathrm{sat}}\left(T_{a}\right)>q_{a}\right]$ at the anemometer level, then the updraft base temperature is computed as the wet bulb temperature corresponding to $q_{a}$. Convection was allowed to affect all unstable layers plus the level immediately above, although if this highest level was at or above the tropopause, then only the unstable levels were deemed to be affected.

Another difficulty was computational instability. This developed due to the leap-frog scheme of moving from time $\tau-1$ to $\tau+1$, if we base the criteria for convective adjustment on data at time $\tau$. This instability caused the solutions to diverge and was eliminated (analogously to diffusion schemes) by basing the criteria for convection on $\tau-1$ profiles.

A third difficulty arose in that profiles could become supersaturated. This itself did not immediately appear deliterious; however, when convection ceases, this can cause a sudden increase in precipitation, thus setting up gravity waves. Consequently, supersaturation existing after convection was removed by condensation with latent heat release. A variation on the above, which only seems to arise at the equatorial wall, was when a strong low-level inversion occurs. This allows the mixing ratio of the environment to be appreciably greater than the mixing ratio of the updraft. In this case we hold the environment mixing ratio constant during convection. All condensate in the updraft contributes to precipitation and the colder updraft cools the environment. This effectively removes the inversion with time.

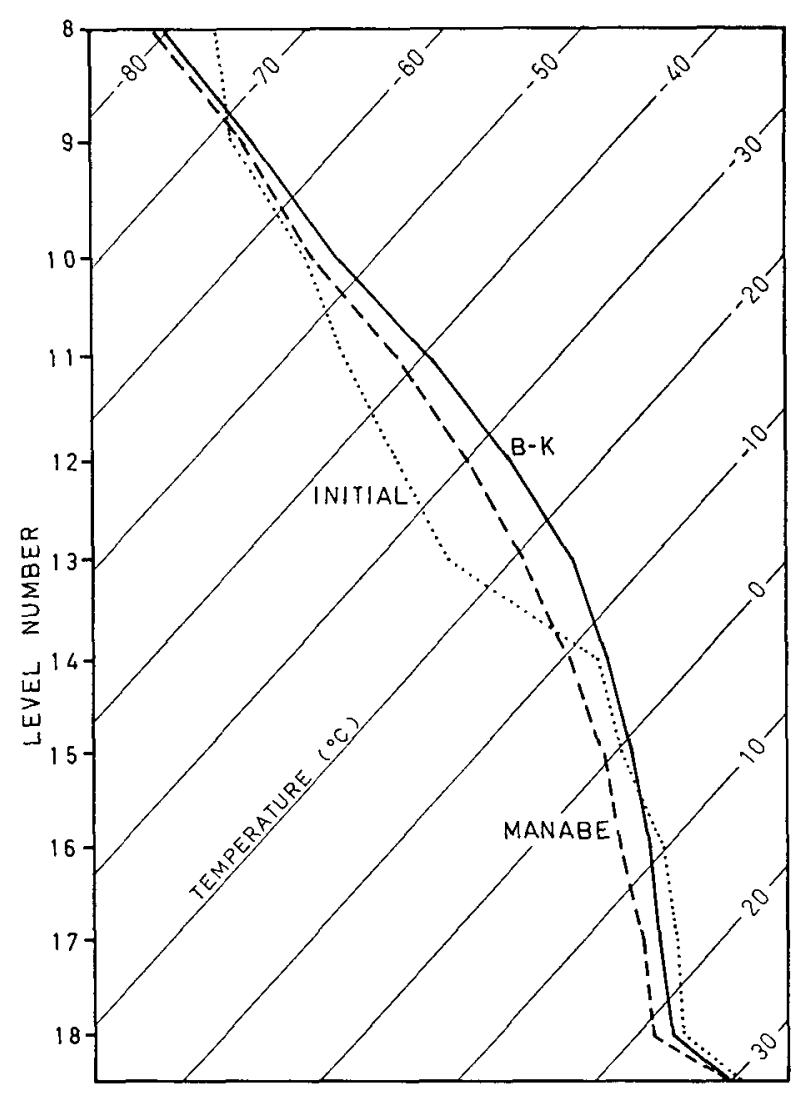

FIG. 3a. The temperature profile for an active tropical point compared with profiles $10 \mathrm{hr}$ later when the $\mathrm{B}-\mathrm{K}$ and $\mathrm{M}$ schemes were used.

The calculations were made on an IBM $360 / 65$ computer. One time step with a real time of $10 \mathrm{~min}$ took just over 1-min computational time for both the $\mathrm{M}$ and $\mathrm{B}-\mathrm{K}$ calculations.

\section{Results}

Results using the $\mathrm{B}-\mathrm{K}$ scheme were obtained for a real time of $10 \mathrm{hr}$ and compared with results using the M scheme. We shall first discuss in detail the differences in the two convective adjustments for just one time step, and later consider differences after 60 time steps $(10 \mathrm{hr})$. Ten hours adequately covers the life cycle of most convective clouds, and allows us to compare how the two schemes treat both the active and decaying stages of the clouds. At the present time the $\mathrm{B}-\mathrm{K}$ scheme is undergoing trials on real data in a forecast model, and discussion of the interaction between the clouds and the synoptic scale disiurbance will be left to a later date.

The chosen tropical profile presented in Fig. 3a favors convective activity. Strong boundary-layer convergence and marked advection of water vapor exist. The initial relative humidity is $100 \%$ to level 13 , and then falls away (Fig. $3 \mathrm{~b}$ ). We now define changes in temperature 


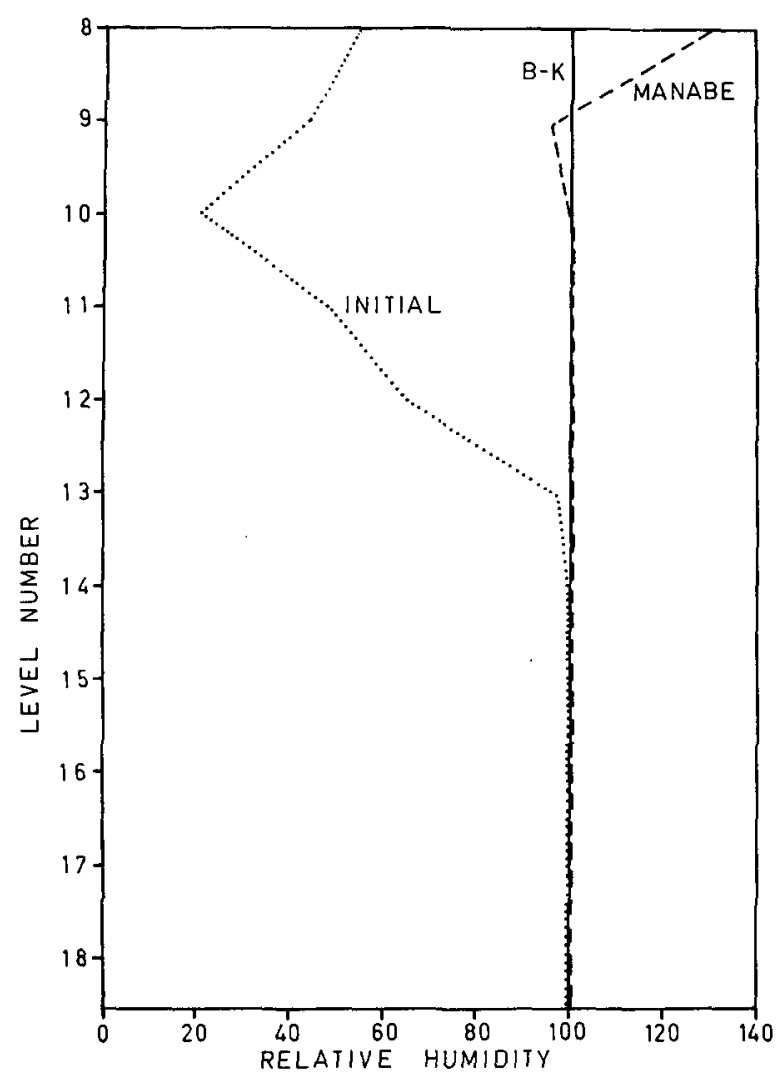

FIG. 3b. As in Fig. 3a except for relative humidity (percent).

$(\Delta T)$ and mixing ratio $(\Delta q)$ as

$$
\left.\begin{array}{rl}
\Delta T & =T^{\tau+1}(\text { adjusted })-T^{\tau-1} \\
\Delta q & \left.=q^{\tau+1} \text { (adjusted }\right)-q^{\tau-1}
\end{array}\right\} .
$$

These are small, and are multiplied by a constant $c$ (equal to the inverse of the boundary layer convergence) for illustrative purposes. Fig. 4a presents $\Delta T$ given by the $\mathrm{B}-\mathrm{K}$ scheme, the $\mathrm{M}$ scheme, and for the purely advective case (which would exist if no convective adjustment were applied). Figs. $4 \mathrm{~b}$ and $4 \mathrm{c}$ present similar profiles for $\Delta q$ and the relative humidity $(h)$, respectively. The $M$ scheme adjusts from the lowest level 18 up to level 13 , removing excess advected water vapor (Fig. 4b) from levels 18,17, 16 and 14, while adding water vapor to levels 15 and 13 . A high rate of precipitation occurs $\left(0.831 \mathrm{~cm} \mathrm{hr}^{-1}\right)$ and the release of latent heat offsets some of the advective cooling in levels 18, 17 and 16, (notice from Fig. 4a that there is still resultant cooling); and it leads to heating in levels 15,14 and 13 . The $\mathrm{B}-\mathrm{K}$ scheme adjusts levels $18-9$. There is a similar rate of precipitation $\left(0.780 \mathrm{~cm} \mathrm{hr}^{-1}\right)$ to $\mathrm{M}$, and excess advected moisture is removed from levels $18-13$ and added to $12-9$. The distribution of heat is similar to $\mathrm{M}$ in that there is cooling in the lower levels and heating in the upper levels, but levels 18, 17 and 16 are not appreciably cooled, latent heat virtually neutralizing the advection. Levels 12 and 11 are warmed in contrast to M. Fig. 4c presents the relative humidities after convective adjustment. If only advection occurred, a super-moist adiabat would have existed. Both adjustments return about a pseudo-adiabatic profile to level 13. The B-K scheme, however, considerably increases the relative humidity in layers 12-9. Consideration of other profiles confirms the general impression obtained from the above discussion.

In general, the $\mathrm{B}-\mathrm{K}$ scheme differs from the $\mathrm{M}$ scheme in that the latter only adjusts saturated unstable layers, whereas the former adjusts through the depth of convection. A result of the $B-K$ scheme is that moisture is added to the environment as cloud matter evaporates. This leads to a noticeable difference in $\Delta q$ between the two schemes. This in turn affects $\Delta T$. The B-K scheme usually cools the lower levels and warms the upper levels through the depth of convection.

Fig. 3a presents the profile considered previously (initial) and after 60 time steps (10 hr) using the $\mathrm{B}-\mathrm{K}$ and $M$ schemes. The final profiles are moist adiabats. Surface temperatures are essentially the same, but the $\mathrm{B}-\mathrm{K}$ profile is a few degrees warmer than the $\mathrm{M}$ profile. Both methods cool the initial profile in the lower levels,

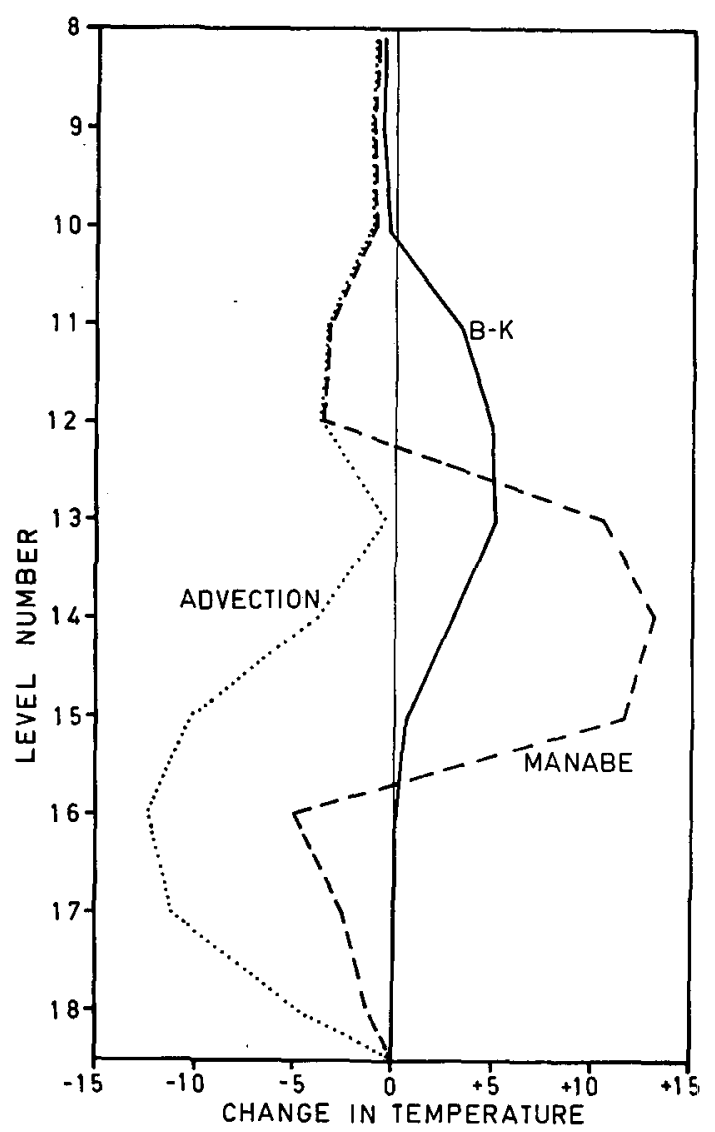

FIg, 4a. Temperature changes (multiplied by $c=10.52$ and in ${ }^{\circ} \mathrm{C}$ ) in going from time step $\tau-1$ to $\tau+1$ allowing only advection, then either using the $B-K$ or the $M$ schemes. 
and heat in the upper levels. The relative humidity, shown in Fig. 3b, confirms that the profiles are saturated, both methods having added considerable moisture to the upper levels. The $M$ relative humidity curve temporarily exceeds $100 \%$ at level 8 .

Fig. 5 presents the temperature profile of another point, which during $10 \mathrm{hr}$ has progressed to a convective situation. The $\mathrm{B}-\mathrm{K}$ scheme has adjusted this point fairly regularly, whereas the $M$ scheme has not adjusted it at all. Hence, the $M$ profile reflects the effect of advection, which is to cool and increase the moisture in the lower levels, while warming and decreasing the moisture in the upper levels. The B-K profile implies that convection modifies the moisture, increasing it all levels. The temperature profile is altered little, and cools less than the $\mathrm{M}$ profile at lower levels. This would eventually lead to a moist adiabat profile which is warmer than the corresponding $M$ profile, in agreement with the point discussed previously. The $\mathrm{B}-\mathrm{K}$ scheme adjusts much earlier than the $M$ scheme, but the $M$ scheme should eventually adjust in a dramatic fashion when the lower levels become saturated.

Several points should be noted when discussing the results. The $\Delta q$ defined in (16) can be considered equal to $b\left(q_{u}-q_{e}+\sigma_{u}{ }^{*}\right) 2 \delta t$ [as obtained from (12)], plus the effect of precipitating out moisture when the relative

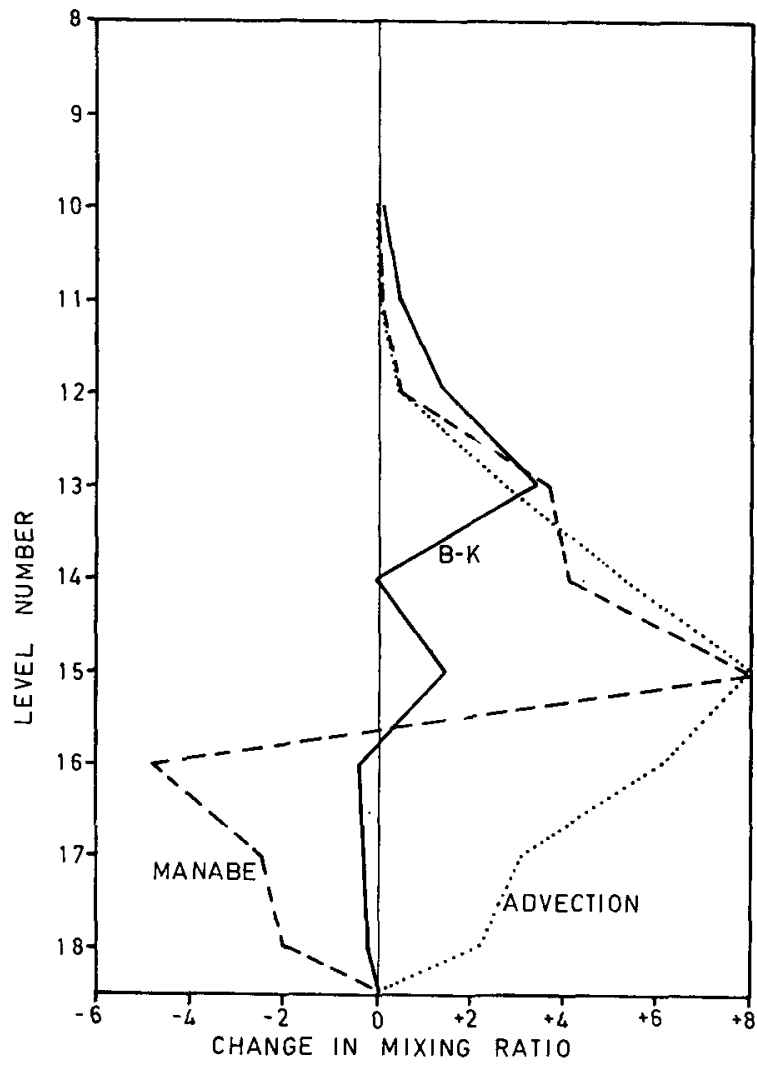

FIG. 4b. As in Fig. 4a except for mixing ratio changes (multiplied by $c=10.52 \times 10^{3}$ and in $\mathrm{gm} \mathrm{kg}^{-1}$ ).

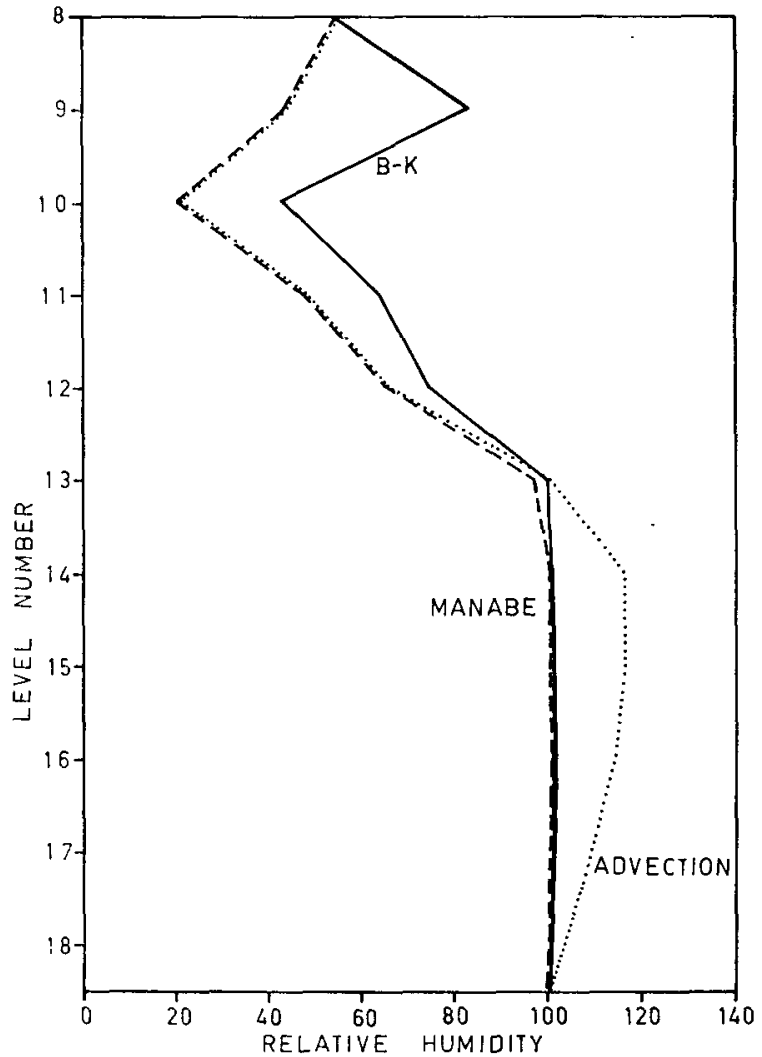

FIg. 4c. As in Fig. 4a except for relative humidity (percent).

humidity is greater than $100 \%$. Similarly, $\Delta T$ of (16) incorporates $b\left[T_{u}-T_{e}-\left(L / C_{p}\right) \sigma_{u}{ }^{*}\right] 2 \delta t+\delta T$ from (12), plus the heating due to excess precipitation. The magnitude of the temperature correction $\delta T$, which is constant with height, is usually of the order of $10 \%$ of the range of $\Delta T$, though in particular situations it can become larger. The principle contribution to $\delta T$ appears to arise from the assumption that $\bar{W}=0$ during convection. The general circulation model includes large-scale motion in the computation of AEC via (9), and hence $\delta T$ from (11) includes broad-scale motion. In practice, this nearly always leads to cooling ( $\delta T$ negative) for convective situations. In Fig. 4a, a $\delta T=-0.7$ is incorporated in $\Delta T$. Further, the B-K scheme is not in equilibrium with the model at this stage, whereas the M scheme is.

A qualitative comparison can be made with the observations of Reed and Recker (loc. cit.). Points which undergo convection for a period of $10 \mathrm{hr}$ may be considered to be located in the trough (T) region defined by Reed and Recker, and final profiles can be compared with their category 5. From their Fig. 5 it can be seen that convective activity cools the atmosphere up to $5 \mathrm{~km}$ (just below level 15), warms from $5-13 \mathrm{~km}$ (level 9) and cools above this. This is in excellent agreement with the results given in Fig. 3a. Further, Fig. 6 of Reed and Recker shows that the relative humidity of 


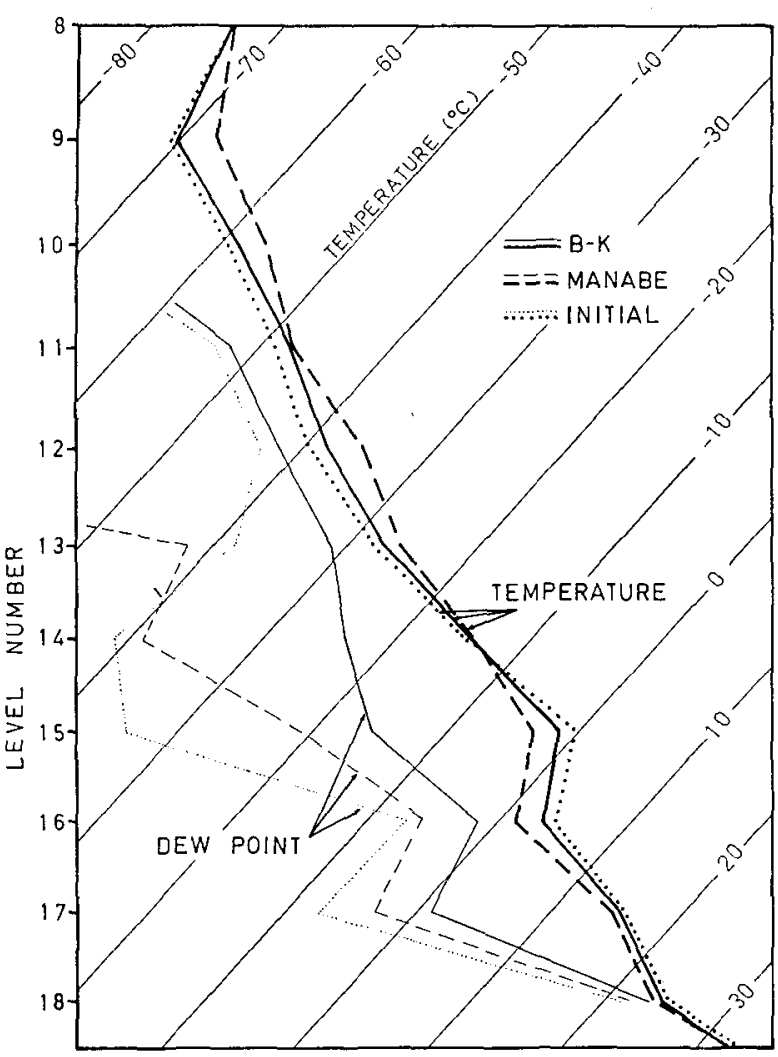

FIG. 5. Temperature and dew point profiles similar to Fig. 3a for a less active point.

the atmosphere increases appreciably, which is what we would expect from the moist adiabat formed in Fig. 3b, and the increase in dew point in Fig. 5. A study of the moisture budget of an active convective point over $10 \mathrm{hr}$ shows that although high rates of evaporation, precipitation and advected moisture are attained, very little moisture is stored at that grid point. This agrees with Table 1 of Reed and Recker, and does not contradict the observations of increased relative humidity because of temperature changes.

Analysis of the longer run also shows that the $\mathrm{B}-\mathrm{K}$ scheme is applied to more than three times the number of grid points compared to the M scheme, and indicates some form of convective cloud over approximately $30 \%$ of the tropics, compared to $6 \%$ for the M scheme. However, strong convection, where the convective precipitation rate exceeds $1 \mathrm{~cm} \mathrm{day}^{-1}$, occurs over only $7 \%$ of the tropics $(M=5 \%)$. These figures agree well with charts of cumulus and cumulonimbus clouds shown in the Atlas of Climatic Charts of the Oceans [U. S. Government Printing Office (1938); see also Riehl and Malkus (1958)]. It is noticeable that the $M$ scheme adjusts frequently (although the adjustments are usually small in the lower layers) in the middle and high latitudes. The B-K scheme adjusts little in the mid-latitudes, and very rarely at high latitudes. In mid-latitudes the B-K adjustment is usually only over a small number of levels, and al though the hydrometeor profile $\sigma$ is chosen for a tropical cumulus cloud, the B-K adjustment seems to yield at least as realistic a change as the $M$ adjustment.

\section{Conclusions and future research}

The results of this study show the ability of a parameterization of a cloud model to maintain the stability of the model atmosphere. In a similar manner to convective clouds the model clouds add excess heat of the updraft to the upper troposphere and reduce the heat of the lower troposphere by evaporation of cloud matter. In both the real atmosphere and the model atmosphere, it is the interaction of the clouds with the broad-scale flow which maintains stability.

The $\mathrm{B}-\mathrm{K}$ cloud model parameterization for convection has been compared with the $\mathrm{M}$ lapse rate adjustment method and found to tend toward a similar pseudo-adiabatic profile. Advantages of the $\mathrm{B}-\mathrm{K}$ scheme are as follows.

(i) There is an early onset of convection in the unsaturated environment.

(ii) The adjustment proceeds regularly and the initial thermodynamic shock induced by sudden adjustment at saturation is eliminated.

(iii) The $B-K$ system always operates through the full depth of convection and leads to smooth profiles.

(iv) Moisture is explicitly redistributed in the vertical.

The $\mathrm{B}-\mathrm{K}$ parameterization retains the energy and moisture conservation properties of the $\mathrm{M}$ scherne, while presenting a more physically realistic model of convective clouds.

The empirically derived quantity required in the $\mathrm{B}-\mathrm{K}$ parameterization is the updraft hydrometeor concentration $\sigma_{u}$. This quantity is intimately related to the microphysics of a cloud and must be expected to vary between clouds. In the context of representing a statistical ensemble of cumulonimbus clouds, the profile used has many desirable features. The concentration initially increases with height corresponding to the increasing buoyancy of the updraft; the initial rate of increase is near the adiabatic value for tropical air; and there is a decrease toward the high troposphere as the air density and frictional drag decrease. The profile compares favorably with theoretical calculations of other researchers. In the integration it was found that the parameterization was most frequently invoked in tropical regions. This substantiates our choice of a tropical $\sigma_{u}$ but if necessary the parameterization could be extended to include a variety of $\sigma_{u}$ profiles for different convective clouds. In practice, it was found that the model was not sensitive to the magnitude of $\sigma_{u}$. A trial computation showed that if a larger $\sigma_{u}$ profile was chosen, then the rate of efflux was smaller. Thus, the 
effect on the environment of a small number of clouds with a large hydrometeor profile was comparable with that of a larger number of clouds with a small hydrometeor profile. The fact that $\sigma_{u}$ was near the adiabatic value at low levels (which are the only ones adjusted at mid latitudes) leads to a surprisingly good adjustment at these latitudes.

Consequent on the $\mathrm{B}-\mathrm{K}$ parameterization operating over a larger area of the tropics and in unsaturated air, it is expected that over a longer integration the average humidity of the troposphere will increase. From this limited run of $10 \mathrm{hr}$ real time, it appears that the humidity may be increasing rather too much in the mid troposphere, which is probably caused by the assumption of a constant efflux rate $b$ [i.e., compare the detrainment profile of Yanai et al. (loc. cit.) and Ogura and Cho (loc. cit.)]. The distribution of precipitation appears to constitute a marked improvement over that produced using the $\mathrm{M}$ scheme.

Future research using this convection scheme will progress along two lines. First, it will be incorporated into the forecasting model, and hence will operate on real data, enabling comparisons of computed and observed fields, particularly precipitation. Second, the run commenced above will be continued out for tens of days and comparisons made with the observed general circulation. It may be necessary to modify the empirical profiles for these longer runs. It is planned to report the results from these runs at a future date.

Acknowledgments. The authors wish to express thanks to B. Hunt for helpful advice during the incorporation of the $\mathrm{B}-\mathrm{K}$ parameterization within the framework of the general circulation model; and further for making the results of the integration using the $M$ scheme available. They also wish to thank Dr. G. B. Tucker and Mr. R. H. Clarke for useful comments on the manuscript. The calculations were performed at the computing centre of the Commonwealth Bureau of Meteorology, Melbourne. Thanks are also due to Mr. R. Weinert who drafted the figures, and Miss G. Burt who typed the manuscript.

\section{APPENDIX A \\ Derivation of Equations}

The mean rate of change of temperature $T$ at a level due to vertical eddy convergence of energy and associated diabatic processes is given by

$$
\frac{\partial \bar{T}}{\partial t}=\frac{1}{\bar{\rho} C_{p}} \frac{\partial}{\partial z}\left(\rho \overline{W^{\prime} E^{\prime}}\right)+\frac{Q}{C_{p}},
$$

where $W$ is the vertical velocity, $E$ the dry static energy $\left[=C_{p} T+g z\right], Q$ the rate of diabatic heating due to condensation and evaporation, the bar designates an area average, while the prime is the local departure from the area average. $C_{p}$ is the specific heat at constant pressure and $\rho$ is the density.
Making the assumption that $\bar{W}=0$, and that the compensating downdrafts are also localized within the cloud, we obtain from (A1)

$$
\frac{\partial \bar{T}}{\partial t}=\frac{1}{\bar{\rho} C_{p}} \frac{\partial}{\partial z}\left[\rho_{u} A_{u} W_{u}\left(E_{u}-\bar{E}\right)+\frac{A_{u} Q_{e}}{C_{p}}+A_{e} \frac{Q_{e}}{C_{p}}\right] .
$$

Here $A_{u}$ is the fraction of the area occupied by the updraft, the subscripts $u$ and $e$ refer to the updraft and the environment, respectively, $Q_{c}$ is the rate of heating per unit area by condensation within the updraft, and $Q_{e}$ is the rate of cooling per unit area by evaporation in the environment.

The updraft is assumed to occupy only a small fraction of the grid area. Because the updraft energy and the environment energy are not very different, the mean energy can be approximated by the environment energy at that level. Eq. (A2) can be rewritten

$$
\begin{aligned}
& \frac{\partial \bar{T}}{\partial t}=-\frac{\rho_{u} A_{u} W_{u}}{\bar{\rho} C_{p}} \frac{\partial E_{u}}{\partial z}+\frac{\rho_{u} A_{u} W_{u}}{\bar{\rho} C_{p}} \frac{\partial E_{e}}{\partial z} \\
& (1) \quad(2) \\
& -\frac{\left(E_{u}-E_{e}\right)}{\bar{\rho} C_{p}} \frac{\partial}{\partial z}\left(\rho_{u} A_{u} W_{u}\right)+\frac{A_{u} Q_{c}}{C_{p}}+\frac{A_{e} Q_{e}}{C_{p}}
\end{aligned}
$$

where the first term on the right is the adiabatic cooling by the vertical advection within the updraft. With a steady-state updraft this cooling is balanced by the condensation heating within the updraft, term (4). Term (2) is the rate of change of energy of the environment as seen by the ascending updraft, and term (3) is the rate of change of temperature due to the convergence of high energy air within the updraft.

Term (2) of (A3) is made suitable for interpretation by invoking conservation of mass at a level, i.e.,

$$
\rho_{u} A_{u} W_{u}=-\rho_{e} A_{e} W_{e} ;
$$

thus term (2) can be written

$$
\frac{\rho_{u} A_{u} W_{u}}{\bar{\rho}} \frac{\partial E_{e}}{\partial z}=-\frac{\rho_{e}}{\bar{\rho}} A_{e} W_{e} \frac{\partial E_{e}}{\partial z} .
$$

The right-hand term of (A5) contributes to heating due to subsidence, which we assume is negligible compared to downdrafts within the cloud.

Assuming that the geopotential does not vary over the grid area, (A3) reduces to

$$
\frac{\partial \bar{T}}{\partial t}=\frac{A_{e} Q_{e}}{C_{p}}-\frac{\left(T_{u}-T_{e}\right)}{\bar{\rho}} \frac{\partial}{\partial z}\left(\rho_{u} A_{u} W_{u}\right) .
$$

The mean rate of change of mixing ratio $q$ at a level due to vertical eddy convergence of moisture and the convergence of condensation by viscous drag and 
gravitational forces $S$, can be written as

$$
\frac{\partial \bar{q}}{\partial t}=-\frac{1}{\bar{\rho}} \frac{\partial}{\partial z}\left(\rho \overline{W^{\prime} q^{\prime}}\right)+S .
$$

Expanding (A7) gives

$$
\frac{\partial \bar{q}}{\partial t}=-\underset{\bar{\rho}}{\frac{1}{\partial z}} \frac{\partial}{-}\left[\rho_{u} A_{u} W_{u}\left(q_{u}-\bar{q}\right)+A_{u} S_{u}\right]
$$

again assuming $\bar{W}=0$. Here the convergence of condensate by viscous drag and gravitational forces occurs entirely in the updraft and is denoted $S_{u}$.

Again considering only a small fractional area occupied by the updraft, the mean mixing ratio is approximated by the environmental mixing ratio at that level. In this case we can write (A8) as

$\frac{\partial \bar{q}}{\partial t}=-\frac{\rho_{u}}{\bar{\rho}} A_{u} W_{u} \frac{\partial}{\partial z} q_{u}+\frac{\rho_{u} A_{u} W_{u}}{\bar{\rho}} \frac{\partial}{\partial z} q_{e}$

$$
-\frac{\left(q_{u}-\bar{q}\right)}{\bar{\rho}} \frac{\partial}{\partial z}\left(\rho_{u} A_{u} W_{u}\right)+A_{u} S_{u} \text {. }
$$

Term (2) of (A9) corresponds to term (2) of (A3) and can be treated in a similar manner. However, the term that is now neglected is a small term which contributes to drying the atmosphere.

Term (1) of (A9) is the vertical advection of moisture in the updraft. The updraft is in a steady state and saturated. This vertical advection is directly related to the rate of condensation at a level. However, due to viscous drag and gravitational forces, the condensate within the updraft is redistributed. Let us define $\sigma_{u}$ as a steady-state condensate concentration at a level representing the balance between the rate of condensation and the condensate convergence in the vertical. Thus, using mass convergence, we have

$$
-\frac{\sigma_{u}}{\bar{\rho}} \frac{\partial}{\partial z}\left(\rho_{u} A_{u} W_{u}\right)=A_{u} S_{u}-\frac{\rho_{u} A_{u} W_{u}}{\bar{\rho}} \frac{\partial q_{u}}{\partial z} .
$$

We can further divide $\sigma_{u}$ into the condensate which is evaporated to the environment $\sigma_{u}{ }^{*}$, and that condensation which is ejected from the updraft as precipitation $\left(\sigma_{u}-\sigma_{u}{ }^{*}\right)$, i.e.,

$$
\begin{aligned}
& -\frac{\sigma_{u}}{\bar{\rho}} \frac{\partial}{\partial z}\left(\rho_{u} A_{u} W_{u}\right) \\
& =-\left(\frac{\sigma_{u}-\sigma_{u}{ }^{*}}{\bar{\rho}}\right) \frac{\partial}{\partial z}\left(\rho_{u} A_{u} W_{u}\right)-\frac{\sigma_{u}{ }^{*}}{\bar{\rho}} \frac{\partial}{\partial z}\left(\rho_{u} A_{u} W_{u}\right) .
\end{aligned}
$$

Since the precipitation does not contribute to $\partial \bar{q} / \partial t$, (A9) reduces to

$$
\frac{\partial \bar{q}}{\partial t}=-\frac{\left(\sigma_{u}^{*}+q_{u}-q_{e}\right)}{\bar{\rho}}-\frac{\partial}{\partial z}\left(\rho_{u} A_{u} W_{u}\right) .
$$

Simplification of (A12) and (A6) is introduced by relating the mass convergence in the updraft to the mass efflux rate $b$ from the updraft to the environment, i.e.,

$$
b=-\frac{1}{\bar{\rho}} \frac{\partial}{\partial z}\left(\rho_{u} A_{u} W_{u}\right) .
$$

Eqs. (A6) and (A12) are related thermodynamically by the cooling of the environment due to evaporation of condensate, i.e.,

$$
-L b \sigma_{u}^{*}=A_{e} Q_{e}
$$

where $L$ is latent heat of condensation.

In terms of grid-point values at a level, then (A6) and (A12) for vertical eddy convergence reduce to

$$
\left.\begin{array}{l}
\frac{\partial T}{\partial t}=b\left(T_{u}-T_{e}-\frac{L}{C_{p}} \sigma_{u}^{*}\right) \\
\frac{\partial q}{\partial t}=b\left(\sigma_{u}{ }^{*}+q_{u}-q_{e}\right)
\end{array}\right\} .
$$

\section{APPENDIX B}

\section{Choice of the Parameter $a$}

As condensation occurs within the updraft, vertical motions redistribute the condensate. Within the updraft the condensate profile is considered to be in a steady state.

It is necessary to delineate the effiux of condensate from the updraft into that part which contributes to precipitation and that part which is evaporated to the environment. Clearly, if the environment is saturated all the efflux of condensate from the updraft will contribute to precipitation. Also, the evaporation rate of the condensate from the updraft will be high if the environment humidity is low. Under this latter condition very little condensate will appear as precipitation.

The measurements used by Kininmonth were made around isolated large convection systems over northeastern Venezuela, a tropical continental locality. Typically the tropospheric environment was near 50\% relative humidity and, although the individual clouds were precipitating, the area average contribution to precipitation was negligible. Consequently, for this study the computed updraft condensate profile of Kininmonth was taken as the maximum and it was assumed that at $50 \%$ relative humidity all condensate is evaporated. A linear relationship between $50 \%$ relative humidity and saturation is assumed for the parameter $a$. 


\section{REFERENCES}

Arakawa, A., A. Katayama and Y. Mintz, 1968: Numerical simulation of the general circulation of the atmosphere. Proc. WMO-IUGG Symp. Numerical Weather Predictions, Vol. 4, Tokyo, 7 to $8-12$.

Bates, J. R., 1972: Tropical disturbances and the general circulation. Quart. J. Roy. Meteor. Soc., 98, 1-16.

Benwell, G. R., and F. H. Bushby, 1970: A case study of frontal behaviour using a 10 -level primitive equation model. Quart. $J$. Roy. Meleor. Soc., 96, 287-296.

Bunker, A. F., 1971: Energy transfer and tropical cell structure over the central Pacific. J. Atmos. Sci., 28, 1101-1116.

Charney, J. G., and A. Eliassen, 1964: On the growth of the hurricane depression. J. Atmos. Sci., 21, 68-75.

Elsberry, R. L., and E. J. Harrison, Jr., 1972: Effects of parameterization of latent heating in a tropical prediction model. J. Appl. Meteor., 11, 255-267.

Kininmonth, W. R., 1970: Thermal modification of the troposphere due to convective interaction. Atmos. Sci. Paper No. 167, Colorado State University.

Krishnamurti, T. N., and W. J., Moxim, 1971: On parameterization of convective and non-convective latent heat release. J. Appl. Meteor., 10, 3-13.

Kuo, H. L., 1965: On formation and intensification of tropical cyclones through latent heat release by cumulus convection. J. Atmos. Sci., 22, 40-63.

Malkus, J. S., 1963: Tropical convection: Progress and outlook. Proc. Conf. Tropical Meteorology, Rotorua, New Zealand Meteor. Service, 247-277.

Manabe, S., and B. G. Hunt, 1968: Experiments with a stratospheric general circulation model: I. Radiative and dynamic aspects. Mon. Wea. Rev., 96, 477-539.

- - J. Smagorinsky and R. F. Strickler, 1965: Simulated climatology of a general circulation model with a hydrologic cycle. Mon. Wea. Rev., 93, 769-798.

Nitta, T., 1972: Energy budget of wave disturbances over the Marshall Islands during the years of 1956 and 1958. J. Meteor. Soc. Japan, 50, 71-84.
Ogura, Y., 1972: Cumulus modelling and parameterization. Rev. Geophys. Space Phys. (in press).

-.- , and T. Takahashi, 1971: Numerical simulation of the life cycle of a thunderstorm cell. Mon. Wea. Rev., 99, 895-911.

- $\longrightarrow$, and H. Cho, 1973: Diagnostic determination of cumulus cloud populations from observed large scale variables. Preprint, Lab. for Atmos. Res., University of Illinois.

Ooyama, K., 1969: Numerical simulation of the life cycle of tropical cyclones. J. Atmos. Sci., 26, 3-40.

Pearce, R. P., and H. Riehl, 1968: Parameterization of convective heat and momentum transfer suggested by analysis of Carribean data. Proc. W.M.O. I.U.G.G. Symp. Numerical Weather Prediction, Tokyo, Japan Meteor. Agency, 175-184.

Reed, R. J., and E. E. Recker, 1971: Structure and properties of synoptic-scale wave disturbances in the equatorial western Pacific. J. Atmos. Sci., 28, 1117-1133.

Riehl, H., 1969: Some aspects of cumulonimbus convection in relation to tropical weather disturbances. Bull. Amer. Meteor. Soc., 50, 587-595.

- - and J. S. Malkus, 1958: On the heat balance in the equatorial trough zone. Geophysica, 6, 503-548.

Rosenthal, S. L., 1970: A circularly symmetric primitive equation model of tropical cyclone development containing an explicit water vapour cycle. Mon. Wea. Rev., 98, 643-663.

Squires, P., and J. S. Turner, 1962: An entraining jet model of cumulonimbus updrafts. Tellhs, 14, 422-434.

Warner, J., 1970: On steady-state one-dimensional models of cumulus convection. J. Atmos. Sci., 27, 1035-1040.

Weinstein, A. I., 1968: A numerical model of cumulus dynamics and microphysics. Ph.D. thesis, Pennsylvania State University.

Yanai, M., 1963: A preliminary survey of large-scale disturbances over the tropical Pacific region. Geofis. Intern., 3, 73-84.

_- S. Esbensen and J. Chu, 1972: Determination of bulk properties of tropical cloud clusters from large-scale heat and moisture budgets. Preprint, Dept. of Meteorology, University of California, Los Angeles.

Zipser, E. J., 1969: The role of organized unsaturated convective downdrafts in the structure and rapid decay of an equatorial disturbance. J. A ppl. Meteor., 8, 799-814. 